\title{
Saturated Compounds in Heavy Petroleum Fractions
}

\author{
Hendrik Muller* and Qasim Saleem \\ Saudi Aramco Research \& Development Center, Dhahran, Saudi Arabia
}

Supporting Information

Corresponding author:

hendrik.muller@aramco.com 
(a)

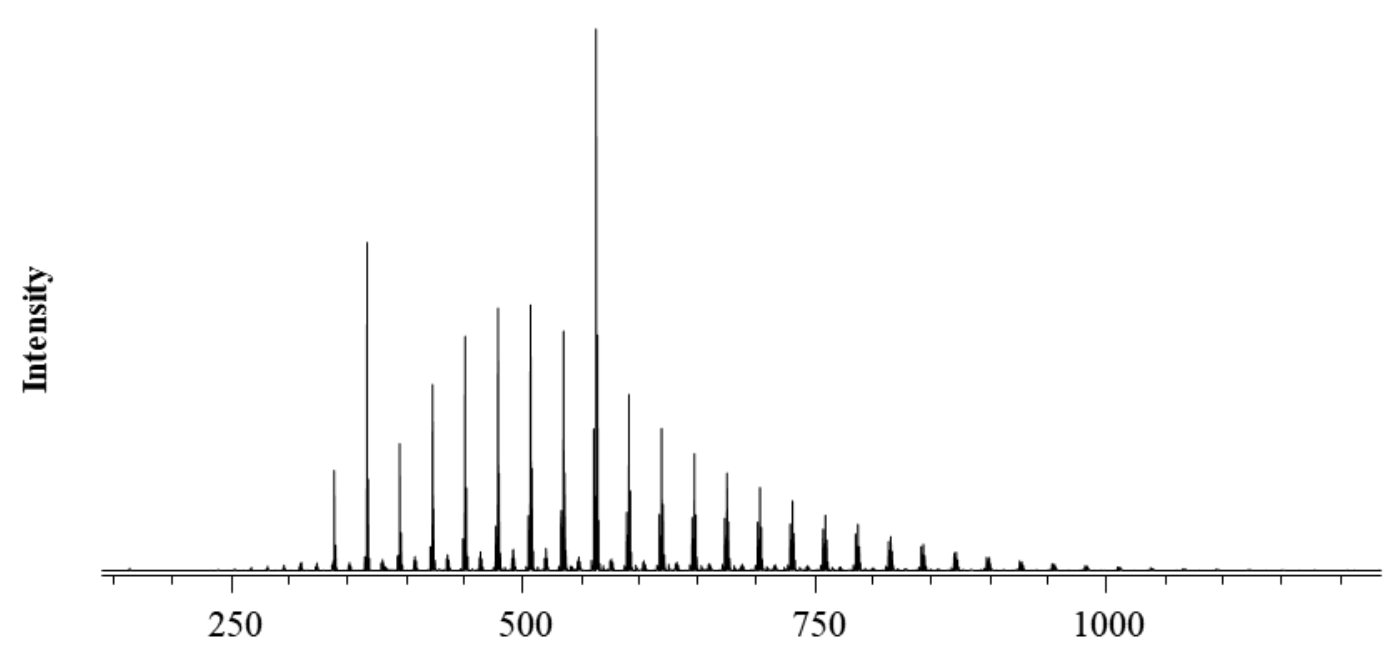

(b)

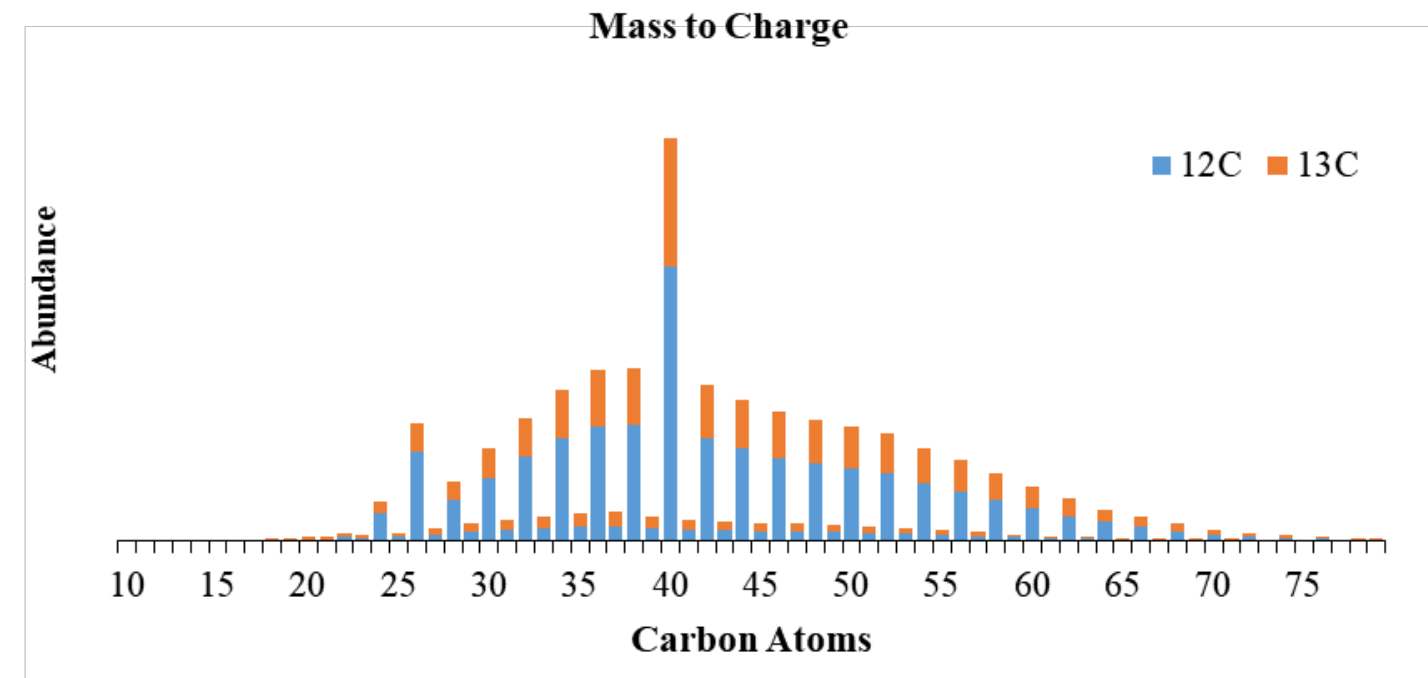

(c)

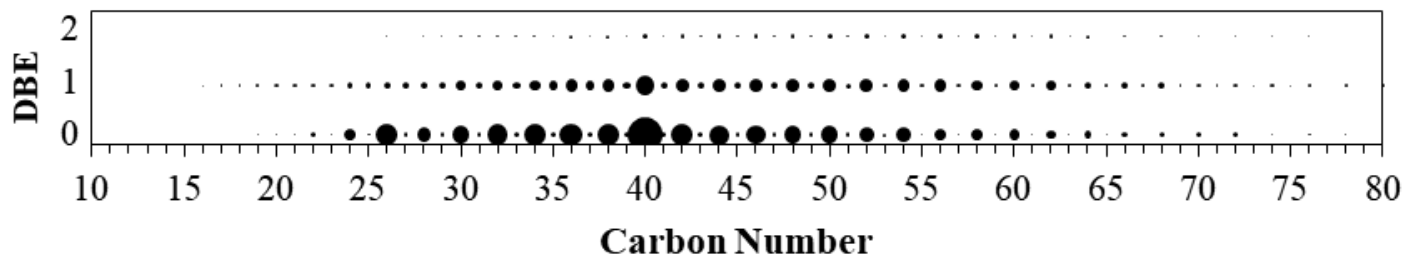

Figure S1. (a) Field desorption time of flight mass spectrum of an n-alkane standard showing alkanes with 20 to 76 carbon atoms. (b) Summed intensity per carbon number with isotope abundances of monoisotopic ions $\left({ }^{12} \mathrm{C}\right.$, blue) and $[\mathrm{M}+1]$ ions $\left({ }^{13} \mathrm{C}\right.$, orange) indicated. (c) Carbon number vs double bond equivalent (DBE) plot showing the paraffins $\mathrm{DBE}=0$, and $\mathrm{M}-2$ and $\mathrm{M}-4$ fragmentation products at $\mathrm{DBE}=1$ and 2, respectively.

The intense signals corresponding to 26 and 40 carbon atoms reflect the higher concentration of those components in the standard. Approximately $20 \%$ fragment ions were observed, consisting mainly of M-2 
and some M-4 ions. Less than $5 \%$ of ions showed a reduction in carbon number, indicated by odd carbon numbers (while the standard mixture consists of even n-alkanes). While the abundance of fragments increased with molecular weight, molecular ions up to n-alkanes with 78 carbon were observed. The light n-alkanes present in the standard, with less than 24 carbon atoms, are lost in the FD measurement.

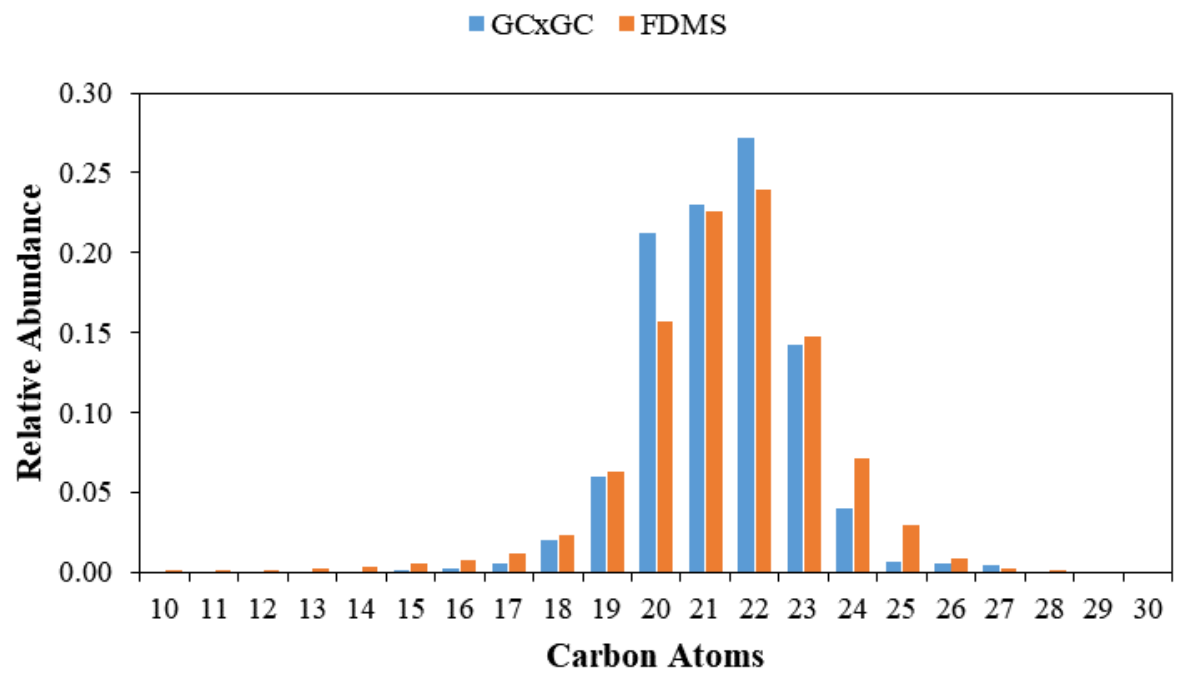

Figure S2. Comparison of carbon number distributions of the saturated compounds identified by GCxGCFID (blue) in the full cut \#14 and the FD TOF MS identified components in the saturates fraction of cut \#14 (orange).

Experimental parameters for comprehensive 2-dimensional gas chromatography (GCxGC) have been reported before,[Muller et al. Energy Fuels 2020, 34, 8260-8273] with flame ionization detector (FID) parameters detailed by Hodgkins et al.,[Hodgkins et al. Industrial \& Engineering Chemistry Research 2019, 58, 19042-19049.] and the chromatographic identification of compounds was based on retention times of standard compounds and literature.[Adam et al. J. Chromatography A 2010, 1217, 1386-139.] 


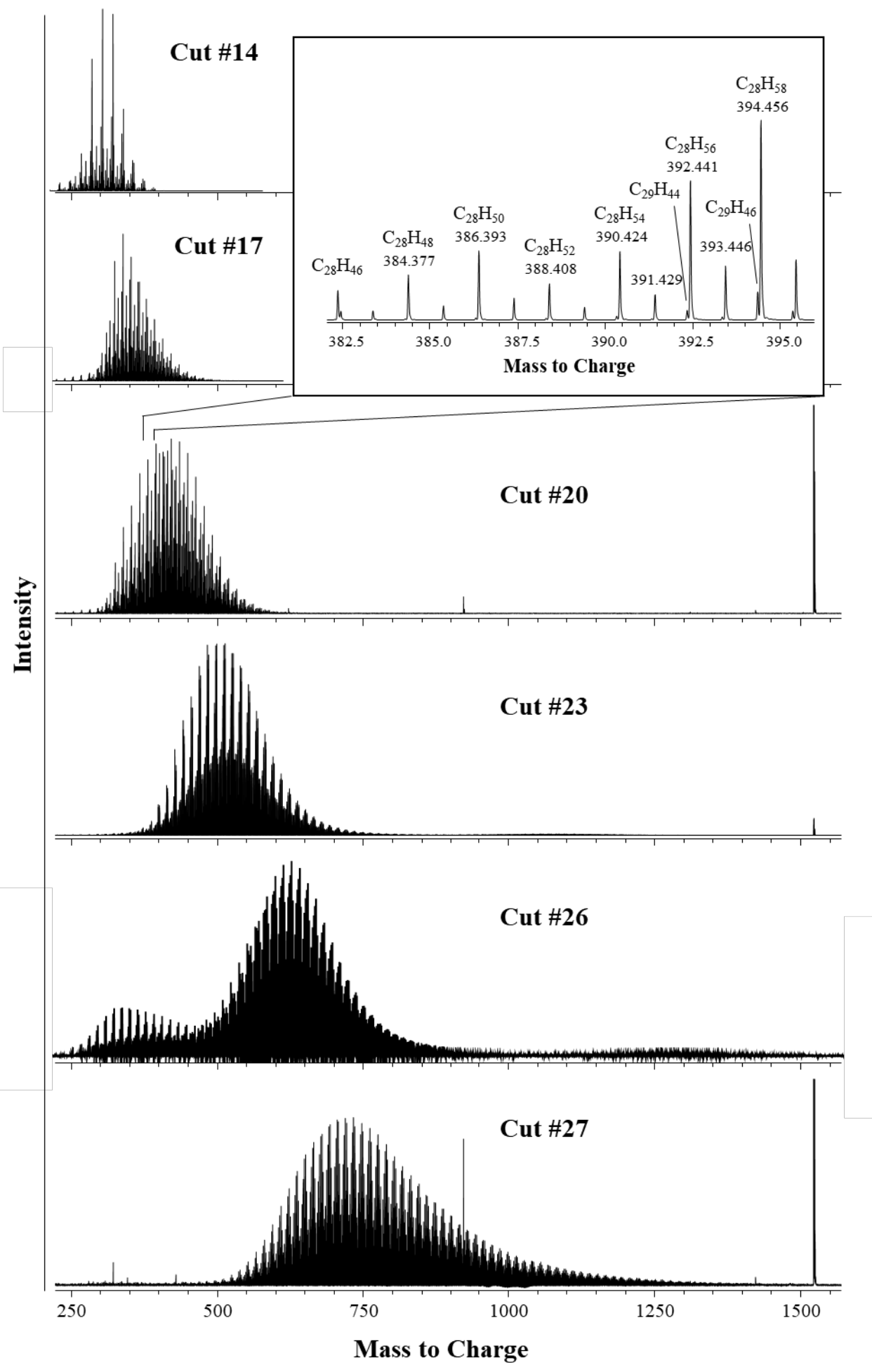

Figure S3. Field desorption time of flight mass spectra showing the increase in molecular weight distribution for the saturates fractions of progressively higher boiling cuts \#14 to \#26, and the corresponding vacuum residue cut \#27. The insert shows an expanded mass region of cut \#20 saturates from $382.4 \mathrm{~m} / \mathrm{z}$ to $395.2 \mathrm{~m} / \mathrm{z}$ with hydrocarbon compounds identified. 

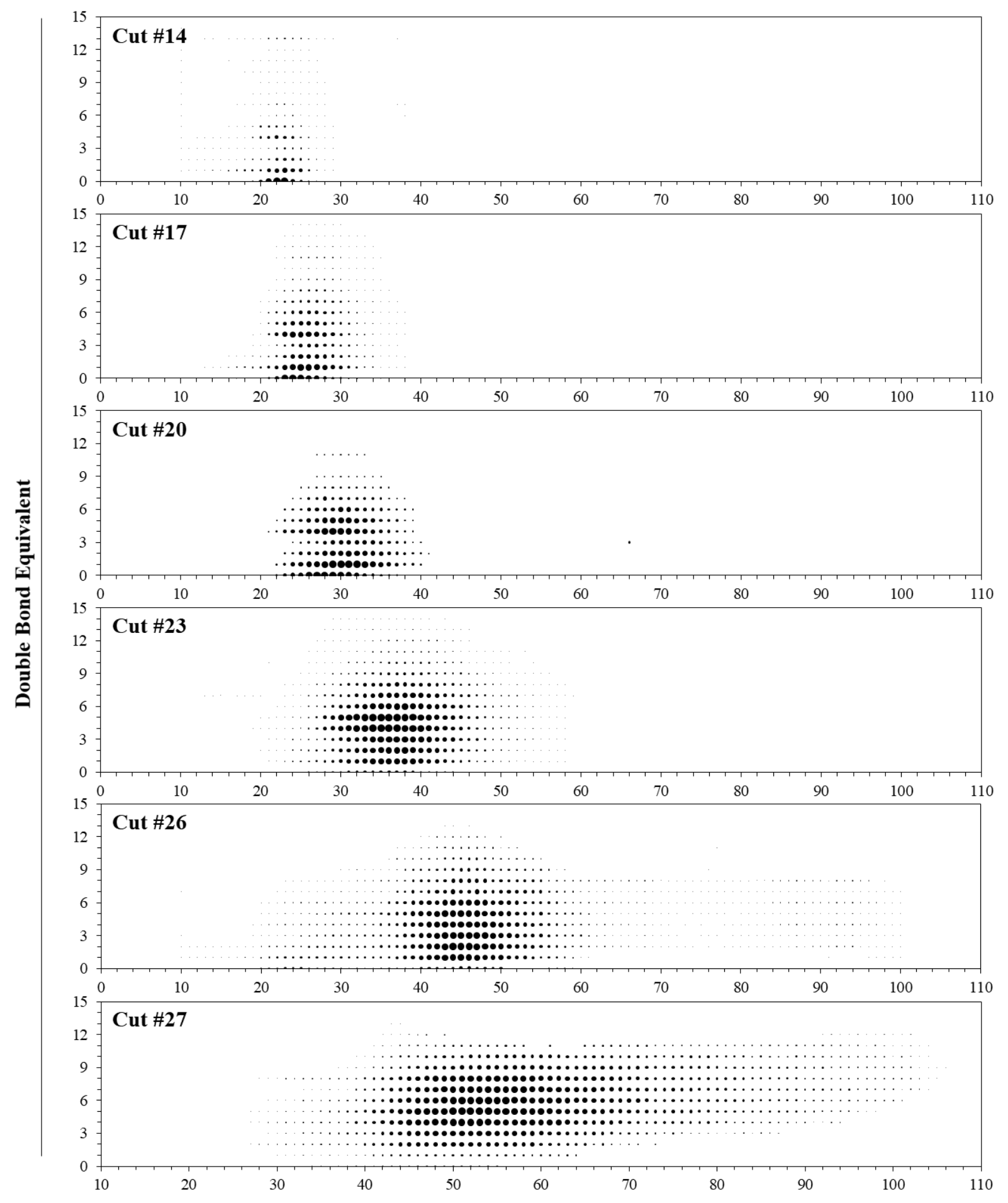

Carbon Number

Figure S4. Identification of hydrocarbon components detected by FD TOF MS in form of DBE vs Carbon number plots. Each dot represents one ion species and its area reflects the mass spectral abundance (including all isotopic signals). The DBE value represents naphthenic rings and/or aromatic rings if DBE $\geq 4$. 
a)

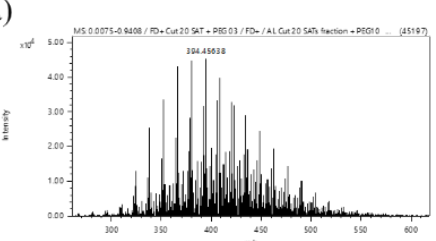

b)

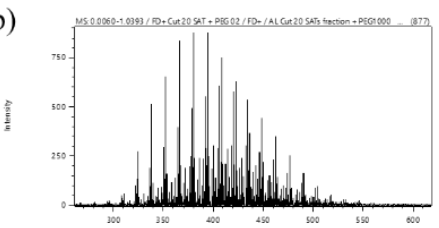

c)

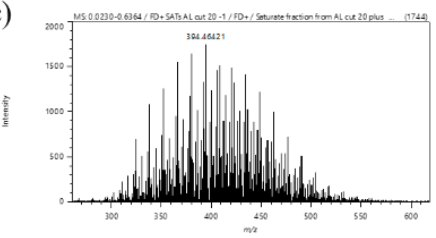

$=12 \mathrm{C}[\mathrm{M}]+=13 \mathrm{C}\left[{ }^{*} \mathrm{M}+\right.$

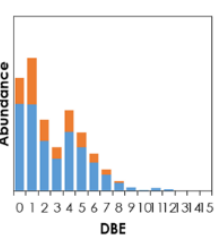

$-12 C[M]+=13 C\left[{ }^{*} M+\right]$
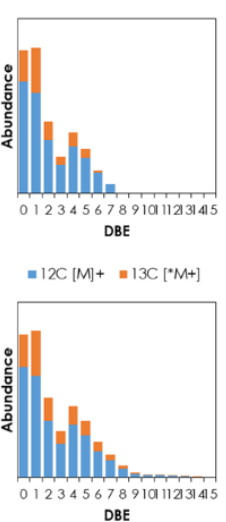
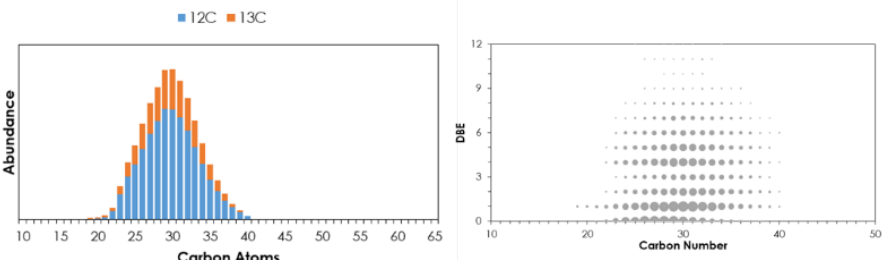

$=12 \mathrm{C}=13 \mathrm{C}$
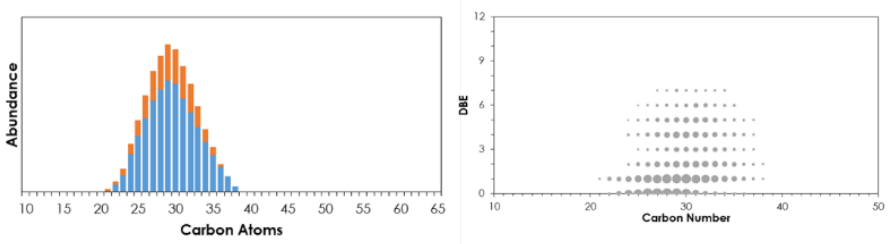

$=12 \mathrm{C}=13 \mathrm{C}$
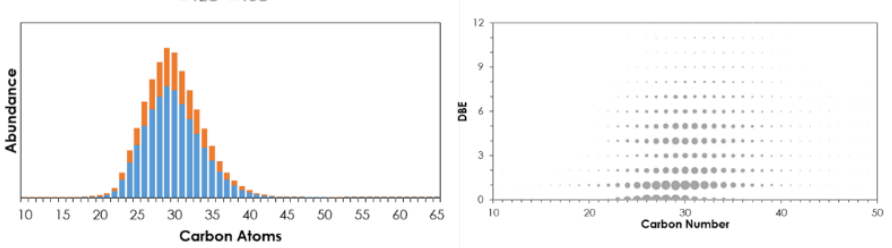

Figure S5. Reproducibility of FD TOF MS identifications; Repeated measurements of the saturates fraction isolated from cut \#20 a) and b), showing from left to right the mass spectrum, DBE and carbon number distributions with 12C isotopes and 13C isotopes distinguished in blue and orange, respectively, and the carbon number vs DBE distribution. The last row c) shows the results for a separately isolated saturates fraction of cut \#20. 

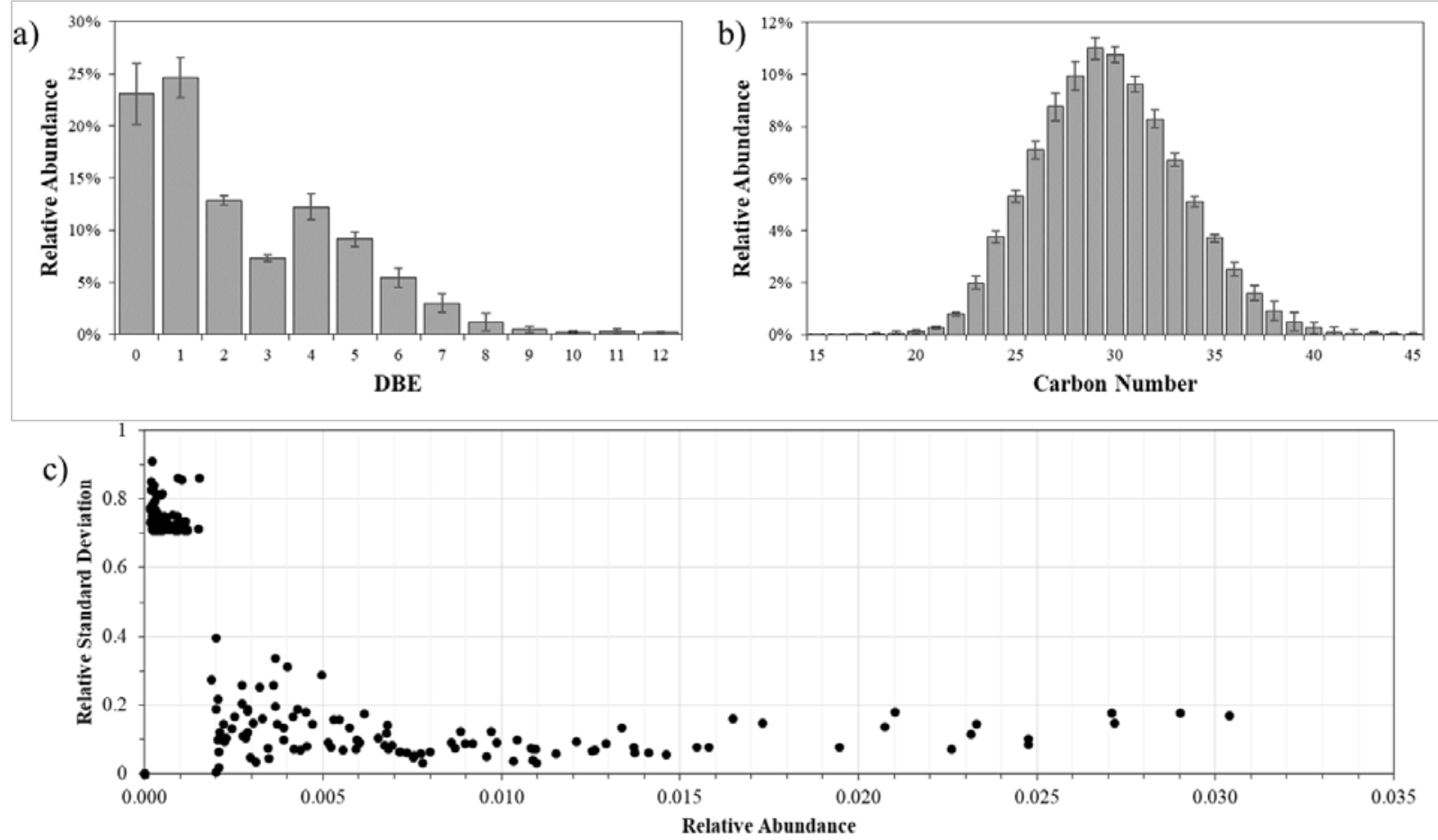

Figure S6. Reproducibility assessment of FD TOF MS data; Standard deviation for 3 repeat measurements of a) DBE lumps, b) carbon number lumps, and c) relative standard deviation of the individual components.

Individual components include the $12 \mathrm{C}$ and $13 \mathrm{C}$ isotope mass signals and show a relative standard deviation below $20 \%$ for components above $0.5 \%$ relative abundance. DBE lumps (including components with different carbon number but the same DBE value) above 5\% relative abundance have a relative standard deviation below 12\%. Carbon number lumps (including components with different DBE values but the same carbon number) above 5\% relative abundance show a relative standard deviation below $7 \%$. Consequently, for all major data points regarding carbon breakdown and DBE series, the relative standard deviation is about $10 \%$ (less than $12 \%$ ) out of three measurements. 


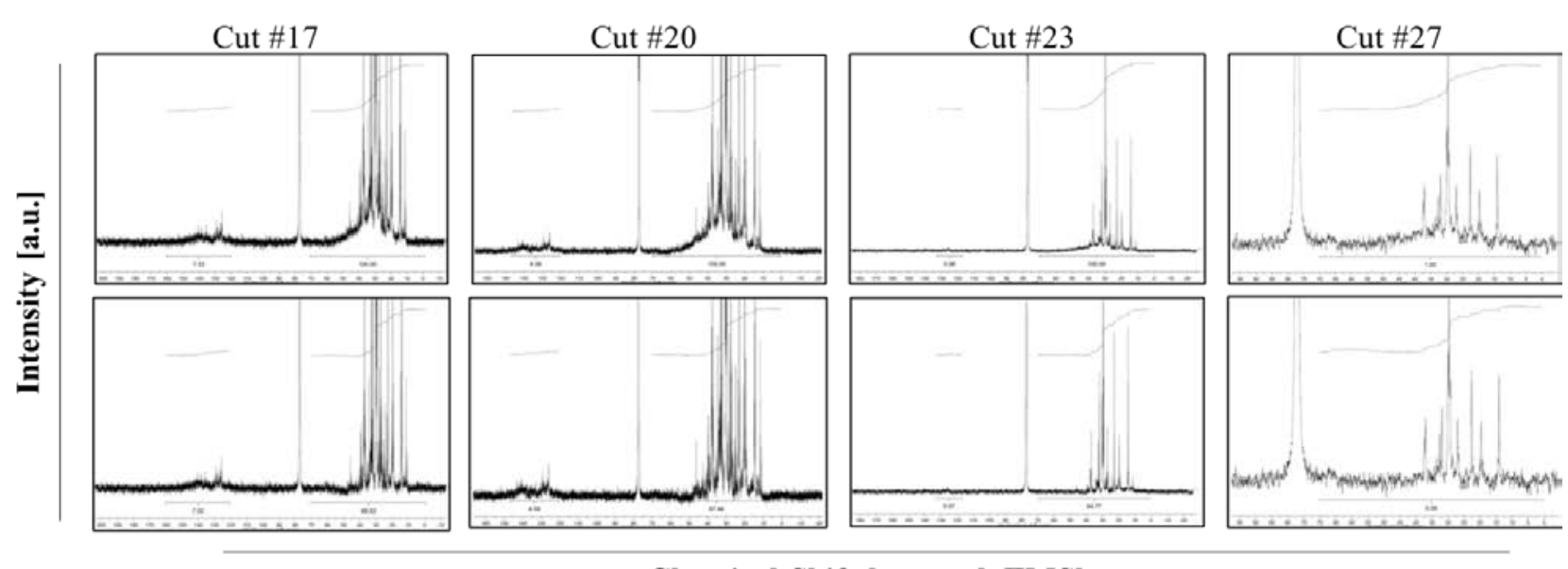

Chemical Shift [ppm rel. TMS]

Figure S7. ${ }^{13} \mathrm{C}$ NMR spectra without baseline adjustment (top row) and after baseline adjustment (bottom row). The baseline adjustment was performed on the region between 20 to $50 \mathrm{ppm}$. The integration difference in the aliphatic region, from 0 to $70 \mathrm{ppm}$ chemical shift, is attributed to naphthenic carbon atoms.

Table S1. Data for calculation of the average number of naphthenic rings per molecule (NR\#). The fraction of carbon atoms in naphthenic rings (C type mole fraction, Table) times the average carbon number is used to calculate the number of aromatic and/or naphthenic rings present in the average molecule. One sample was repeated completely to estimate the experimental reproducibility (saturates fraction isolated from Cut \#20, then measured by NMR and FD TOF MS); Results are included in the bottom row.

\begin{tabular}{cccccc}
\hline Cut & $\begin{array}{c}\text { MW } \\
{[\mathrm{Da}]}\end{array}$ & C\# & Cnap & C\#nap & $\begin{array}{c}\text { NR\# } \\
\text { (NMR \& MW) }\end{array}$ \\
\hline $\mathbf{1 4}$ & 299 & 21.4 & n.d. & n.d. & n.d. \\
$\mathbf{1 7}$ & 362 & 25.9 & 0.293 & 7.6 & 1.4 \\
$\mathbf{2 0}$ & 428 & 30.6 & 0.311 & 9.5 & 1.9 \\
$\mathbf{2 3}$ & 528 & 37.7 & 0.352 & 13.3 & 2.8 \\
$\mathbf{2 6}$ & 628 & 45.6 & n.d. & n.d. & n.d. \\
$\mathbf{2 7}$ & 791 & 56.5 & 0.420 & 23.7 & 5.4 \\
$\mathbf{2 0 ( r e p )}$ & 411 & 29.4 & 0.357 & 10.5 & 2.1 \\
\hline
\end{tabular}

An example NR\# calculation for cut \#17 is as follows: 
For an average MW of $362 \mathrm{Da}$, the corresponding carbon atoms are 25.9 in the average molecule. The mol fraction of $\mathrm{C}($ nap $)=0.293$, times 25.9 carbon atoms per molecule, corresponds to an average of 7.6 carbon atoms in naphthenic rings. An average naphthenic molecule could be hypothesized to contain a six membered core with additional rings formed by four additional carbon atoms. This system could then be described by the following equations:

$$
\begin{gathered}
C(\#) \text { nap }=6+4 n \\
N R \#=1+n \\
\text { Therefore, } N R \#=1+\frac{C(\#) n a p-6}{4}
\end{gathered}
$$

Table S2. Data for calculation of the mass fraction of aromatic molecules based on NMR and MW. The

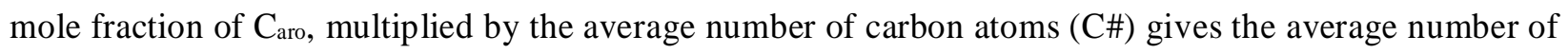
carbon atoms in aromatic rings ( $\mathrm{C \#}$ aro). Assumed that aromatic and saturated molecules have the same average molecular weight, and that only monoaromatic (AR1) compounds are present C\#aro, divided by 6 (carbon atoms make up the one aromatic ring) gives the mass fraction of aromatic compounds. Column AR2 shows the mass fraction of aromatics assumed only diaromatic compounds are entrained (C\#aro divided by 10 carbon atoms that make up the two aromatic rings). AR1 is strongly suggested by the FD TOF MS data.

\begin{tabular}{ccccccc}
\hline Cut & MW & C\# & Caro & C\#aro & AR1 & AR2 \\
& {$[\mathbf{D a}]$} & & & & & \\
\hline $\mathbf{1 4}$ & 299 & 21.4 & n.d. & n.d. & n.d. & n.d. \\
$\mathbf{1 7}$ & 362 & 25.9 & 0.068 & 1.8 & 0.30 & 0.18 \\
$\mathbf{2 0}$ & 428 & 30.6 & 0.044 & 1.3 & 0.22 & 0.13 \\
$\mathbf{2 3}$ & 528 & 37.7 & 0.001 & 0.0 & 0.004 & 0.00 \\
$\mathbf{2 6}$ & 628 & 45.6 & n.d. & n.d. & n.d. & n.d. \\
$\mathbf{2 7}$ & 791 & 56.5 & 0.000 & 0.0 & 0.00 & 0.00 \\
$\mathbf{2 0 ( I I )}$ & 411 & 29.4 & 0.042 & 1.2 & 0.20 & 0.12 \\
\hline
\end{tabular}


The calculation of the aromatic compound mass fraction is now explained on the example of cut \#17 data: The average aromatic and saturated molecules in the cut \#27 saturates fraction have a MW = 362, corresponding to 25.9 carbon atoms. The influence of a slightly lower average DBE value for saturated molecules is ignored in this calculation to reduce the dependency on mass spectrometric identifications. On average, each molecule contains 1.8 carbon atoms in aromatic rings, but assumed either monoaromatic or saturated compounds are present, there can be either molecules with $\mathrm{C} \#_{\text {aro }}=6$, or with $\mathrm{C \#}_{\text {aro }}=0$. Therefore, dividing 1.8 by 6 gives the mol fraction of aromatic compounds, and since they have the same molecular weight, the mol fraction equals the mass fraction.

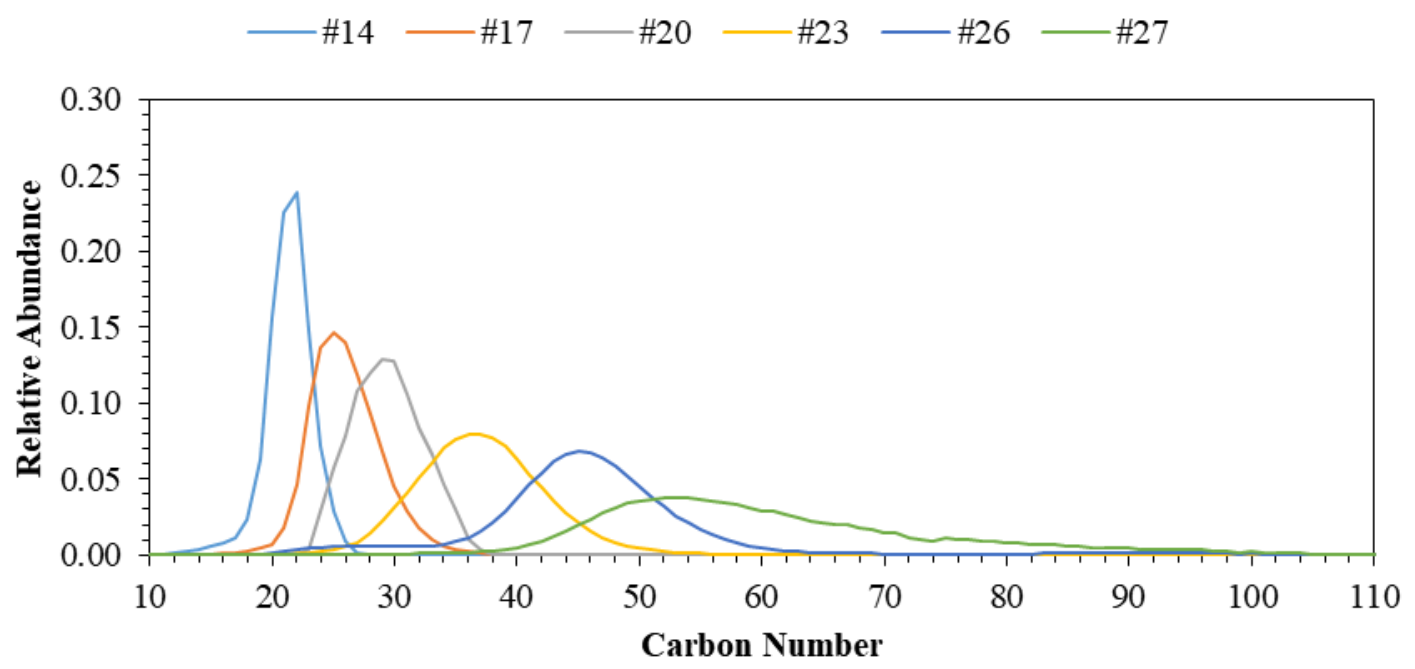

Figure S8. Carbon number distributions obtained by FD TOF MS for the saturated compounds in the indicated cuts. 